\title{
Total Elbow Replacement as an Alternative to Severely Comminuted Fractures around the Elbow
}

\author{
Eyal Ginesin, Doron Norman \\ Department of Orthopedics and Traumatology, Rambam Medical Center, Haifa, Israel \\ Email: E_ginesin@rambam.health.gov.il
}

How to cite this paper: Ginesin, E. and Norman, D. (2017) Total Elbow Replacement as an Alternative to Severely Comminuted Fractures around the Elbow. Open Journal of Orthopedics, 7, 337-344. https://doi.org/10.4236/ojo.2017.711034

Received: August 31, 2017

Accepted: November 5, 2017

Published: November 8, 2017

Copyright $\odot 2017$ by authors and Scientific Research Publishing Inc. This work is licensed under the Creative Commons Attribution International License (CC BY 4.0).

http://creativecommons.org/licenses/by/4.0/

Open Access

\begin{abstract}
Background: Intra articular elbow fractures are considered to be one of the most complex injuries in orthopedic trauma. Some are too comminuted for open reduction and internal fixation. Recently total elbow replacement (TER) had gained popularity for the treatment of comminuted elbow trauma when other treatment options are not possible. Methods: Since 2007-2013 we treated 18 patients with TER due to comminuted distal humeral fractures. We used the Disabilities of the Arm, Shoulder and Hand scoring system (DASH) to evaluate the patient's satisfaction. In addition, we evaluated the elbow range of motion and collateral stability. Results and Conclusions: Functional range of motion was achieved with high patient's satisfaction. Based on the results, we conclude that TER is a reasonable option for complex elbow fractures when open reduction and internal fixation is not suitable.
\end{abstract}

\section{Keywords}

Total Elbow Replacement, Elbow Arthroplasty, DASH Score

\section{Introduction}

Multiple articular fracture of the elbow (Figure 1) is considered to be one of the most complex injuries in the orthopedic trauma. Due to the fact that the elbow is a complex structure that requires precise coordination between three different joints, sometimes the number of broken segments and extensive cartilage damage create a situation in which the attempt to fix the fracture is not applicable or is sub-optimal.

Total elbow replacement (TER) is originally an operative solution for chronic conditions of extensive destruction of the joint, usually as a result of advanced 


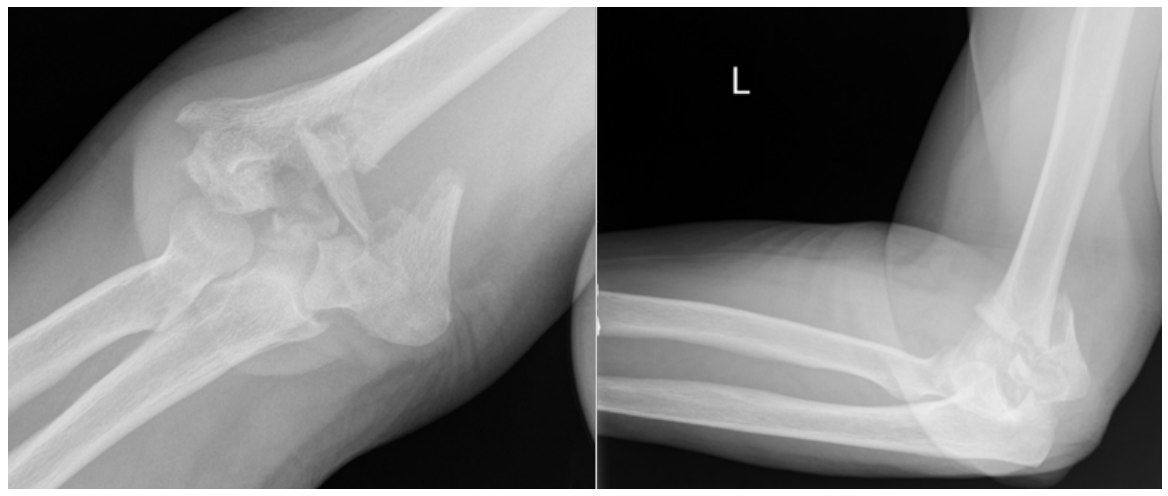

Figure 1. Multiple articular fracture of the elbow (Rambam hospital, orthopedic department).

inflammatory or degenerative changes. In addition, it provides a response in cases of chronic instability of the elbow [1] [2] [3] [4].

During the recent years, with the experience accumulated of the surgical technique, the development of the materials composition, and the structure of the implants, this procedure has also taken its place as an alternative surgery for the treatment of complex fractures of the elbow joint, which cannot be reconstructed and surgically stabilized optimally. This contributes to the relatively quick return to functional life and consequently maintaining the quality of the patient life [5] [6].

\section{The Development and Types of Implants}

TER is based on a two-component implant-a component of the humeral bone and a component of the ulnar bone.

Today the implants are commonly dividing into three main types:

1) Linked/Coupled implant-An implant with a humeral component and an ulnar component that are interconnected.

The first implants produced were constrained implants which were stabilized by using a single axis that allowed for flexion and extension movements only. Over time, many failures have been observed with this implant, due to loosening, especially in the humeral component.

By the time an Unconstrained/Semiconstrained implant, in which the most famous is called Coonrad-Morrey (1973), was developed. It had a feature that allows rotary free movement, Varus and Valgus around $10^{\circ}$, thus reducing the load between the parts of the implant itself and between the implant components and the bone [4] [7] [8]. This structure enables longer life expectancy and stability of the implant [8] [9] [10].

2) Unlinked/uncoupled implant-with time, the understanding of the mechanics and kinematics of the elbow joint was improved. Consequently, the importance of a certain degree of freedom between the implant components and the need for muscle support around the joint was cleared and understood for the efficiency of the implant [7] [11] [12] [13] [14] [15].

The stability of the implant is not only based on the mechanical forces between 
the implanted parts of the implant, but also on the forces acting between the components of the implant to the bone, its position and the adjustment between its components.

This implant creates an anatomical structure close to the native joint between the humeral bone and ulnar bone. The most common types of implants are Souter-Strathclyde and Kudo.

The main advantage of this type of implants is the freedom degree of the joint. This advantage is achieved due to the lower load between the implant parts itself, which reduces the chance of loosening.

Two main disadvantages of this implant are the need for maximum precision between the implant and bone components, as well as a higher incidence of dislocation.

3) Linkable implant-This implant is the new generation and excels the advantages of the two implants described above.

The load on the weight bearing area in these implants is relatively low due to the thick envelope of polyethylene surrounding it. The structure of the implant enables a more accurate anatomical replacement with the option given to the surgeon to decide at the end of the surgery whether to attach the implant components or not.

\section{Anatomical Approaches}

The surgical approaches are based on the incision relative to the triceps muscle.

Splitting the Triceps-the muscle is cut with an inverted V-shape without affecting the attachment of the tendon to the olecranon.

Triceps sparing-This approach allows muscle retention, but the exposure of the elbow joint is very limited and makes surgery difficult.

Shifting the triceps-the triceps muscle is shifting from the inner side to the outer one along with elevating from the proximal humerus and ulna while maintaining continuity with the anconeus muscle without damaging it.

This surgical technique gives a good exposure of the elbow joint and thus obtained the operation performed accurately. At the end of the operation, the muscle is fixed to the ulnar bone through absorbable sutures.

\section{Risks and Complications after TER}

Results of elbow replacement are statistically inferior compared to total hip or knee replacement [5] [16] [17].

Success of TER is proven by achieving the functional range of motion and satisfaction of the patient. The complications rate of elbow replacement after complex fractures ranged between $27 \%$ to $43 \%$ and the rate of postoperative complications ranged from $22 \%$ to $28 \%$ [8] [18] [19].

Typical complications of TER include:

1) Infection-more common than in other joints. This can be attributed to the thin skin tissue in this area. The incidence of infection is between $3 \%$ and $11 \%$.

Treatment of infections is a complex and difficult process that can range from conservative treatment of intravenous antibiotics for weeks to surgical treatment 
that may requires number of repeated operations, but not always with proper success. Implants coated with antibiotics are an effective way to reduce the incidence of infection [10].

2) Decrease extension mechanism - is caused by the quality of the soft tissues surrounding the joint and the surgical manipulations-2\% - 3\%.

3) Aseptic loosening - with the progression of the implants development, this complication decreased significantly but still stands on $2 \%-9 \%$.

Increasing loads on the joint is a risk factor for the loosening of the implant and therefore can be a limitation for young and active patients.

4) Implant wear-is known with each joint replacement. The wear of the implant over time and the free polyethylene parts cause a local inflammatory response that responsible for the osteolysis and osteoporosis of the bone.

5) Periprosthetic fractures-as a result of further injury or loosening of the implant.

6) Dislocation-Unlinked implants tend to dislocate as a result of the implant's dependence on the surrounding soft tissue, as well as the absence of a mechanical connection between the two implant components. The rate of dislocations is currently estimated at $5 \%$. The rate of dislocations and subluxations is estimated at $15 \%$.

7) Ulnar nerve injury-This complication is estimated to be around $5 \%$. In order to avoid this, the nerve must be isolated and shifted during surgery.

\section{Methods}

Between the years 2007 and 2013, we treated 29 patients ( 28 female and 1 male), most of them had complex fractures of the elbow joint (27 cases). The remaining two were operated due to osteoarthritis and progressive joint destruction.

In all surgeries, Biomet's Discovery ( ) elbow system implant was used.

This implant is a semiconstrained, with an axis that allows Varus/Valgus of $7^{\circ}$ using two halves balls moving against of a acetabular plastic structure, giving a large area of contact between the joint (Figure 2).

To date, 18 patients-all female with an average age of 70 years (range 54 - 83) who have been treated after sever elbow injury-have been obtained. Sixteen operations on the right elbow and two on the left. All implants were fixed to the bone using cement material (Table 1).

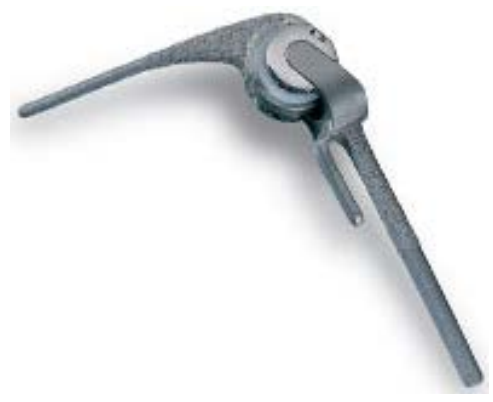

Figure 2. Biomet's Discovery (c) elbow system implant. 
Table 1. Demographic characteristics of the patients.

\begin{tabular}{cc}
\hline Variable & Patients $(\mathrm{N}=18)$ \\
\hline Age at operation (years) & $70.2 \pm 8.3$ \\
Sex male/female & $0(0 \%) / 18(100 \%)$ \\
Side Left/Right & $2(11.1 \%) / 16(88.8 \%)$ \\
Follow up (month) & $26.16 \pm 17.5$ \\
ROM $\left(^{\circ}\right)$ & \\
Flexion & $130 \pm 5.9$ \\
Extension & $-9.4 \pm 11.6$ \\
Pronation & $86.5 \pm 4.7$ \\
Supnation & $81.5 \pm 8.1$ \\
Dash score & $33.1 \pm 25.8$ \\
\hline
\end{tabular}

The duration of follow-up was an average of 26 months during which the satisfaction level was assessed based on a function-based questionnaire-disability of the Arm, Shoulder and Hand score (DASH score) [16]. In addition, the range of motion and collateral stability of the joint were examined.

The elbow collateral stability examination was done with extended elbow, and was determined according to 3 levels-stable, semi-stable, or unstable elbow.

Complications were also followed up such as loosening, infection, wound complications, and nerve damage.

\section{Results}

An analysis of the 18 patients was performed at an average time of 26 months postoperatively and showed satisfaction with an average score of 33 according to DASH score (range 2.5 - 69).

The ranges of motion include flexion and extension with an average of $130^{\circ}$ $\left( \pm 5.9^{\circ}\right)$ in flexion to a lack of extension with an average of $9.4^{\circ}\left( \pm 11.6^{\circ}\right)$. In addition, the supination range was with an average of $81.5^{\circ}\left( \pm 8.1^{\circ}\right)$ and the pronation range was with an average of $86.5^{\circ}\left( \pm 4.7^{\circ}\right)$.

It should be mentioned to say that all the elbows were found to be stable during elbow extension.

Regarding to the complications, there was a single case of aseptic loosening (5.5\%). No cases of neuromuscular or sensory injury were found although a single case of pain attributed to the ulnar nerve was noticed.

\section{Discussion}

With the development of the surgical option for full replacement of the elbow joint, tremendous changes occurred with the types of implants, the materials and the surgical methods.

Those changes can be attributed to the mechanical understanding of the joint complexity that has been learned over the years, the new technology development, and of course the experience accumulated by the surgeon performing this 


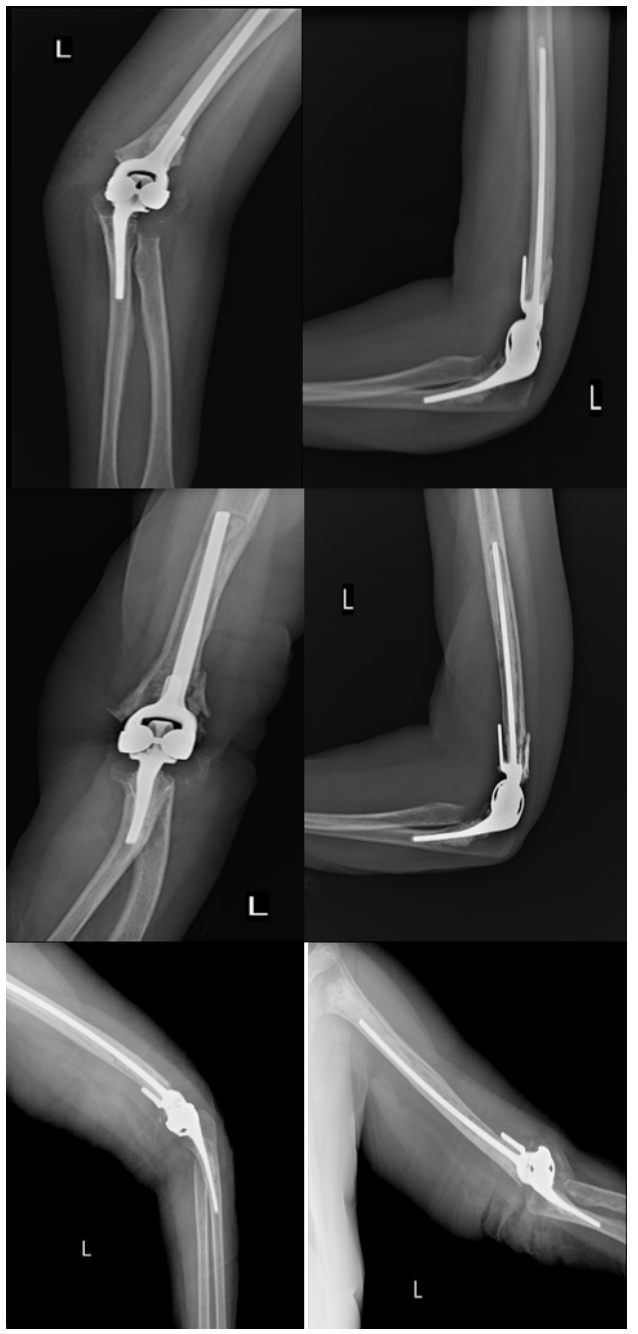

Figure 3. Loosening case of the implant treated with revision TER.

procedure.

In this study, we found a low frequency of complications compared to the known literature. Single loosening case of the implant treated with revision TER resulting return to full function (Figure 3) and a pain disturbance referring to the ulnar nerve without sensory or motor deficit.

Although the incidence of infections in these surgeries is not insignificant, in our study we did not encounter this complication.

In the vast majority of cases, there was a great satisfaction concerning the functional level expressed by the range of motions, absence of significant pain and maintenance of quality of life.

When comparing this study to other studies of TER we can find that the range of motion and satisfactory rate was high and the infection rate was the same.

The limitation of this study can be due to the short follow up and the number of patients.

There are needs of more articles with longer follow up to continue evaluate the effectiveness of this treatment. 


\section{Conclusions}

TER is an effective treatment for specific populations suffering from complex fractures of the elbow when open reduction and internal fixation is sub-optimal.

Over the years, there has been progress in the shape, type and component of implants, as well as in the mechanical understanding of this unique joint that appears to be expressed by the patients' high satisfaction.

\section{Ethical Clearance}

There is no any financial or other benefit to the authors from this study.

\section{References}

[1] Jenkins, P.J., Watts, A.C., Norwood, T., et al. (2013) Total Elbow Replacement: Outcome of 1146 Arthroplasties from the Scottish Arthroplasty Project. Acta Orthopaedica, 84, 119-123. https://doi.org/10.3109/17453674.2013.784658

[2] Plaschke, H.C., Thillenmann, T., Belling-Sørensen, A.K. and Olsen, B. (2013) Revision Total Elbow Arthroplasty with the Linked Coonrad-Morrey Total Elbow Arthroplasty: A Retrospective Study of Twenty Procedures. International Orthopaedics (SICOT), 37, 853-858. https://doi.org/10.1007/s00264-013-1821-9

[3] Athwal, G.S. and Morrey, B.F. (2006) Revision Total Elbow Arthroplasty for Prosthetic Fractures. The Journal of Bone and Joint Surgery-American, 88, 2017-2026.

[4] Kim, J.A., Mudgal, C.S., Konopka, J.F., et al. (2011) Complications of Total Elbow Arthroplasty. Journal of the American Academy of Orthopaedic Surgeons, 19, 328-339. https://doi.org/10.5435/00124635-201106000-00003

[5] Sanchez-Sotelo, J. and Morrey, B.F. (2011) Total Elbow Arthroplasty. Journal of the American Academy of Orthopaedic Surgeons, 19, 121-125.

https://doi.org/10.5435/00124635-201102000-00007

[6] Chalidis, B., Dimitriou, C., Papadopoulos, P., et al. (2009) Total Elbow Arthroplasty for the Treatment of Insufficient Distal Humeral Fractures. A Retrospective Clinical Study and Review of the Literature. Injury, 40, 582-590.

[7] Risung, F. (1997) The Norway Elbow Replacement. Design, Technique and Results after Nine Years. The Journal of Bone and Joint Surgery. British Volume, 79, 394-402.

[8] Choo, A. and Ramsey, M.L. (2013) Total Elbow Arthroplasty: Current Options. Journal of the American Academy of Orthopaedic Surgeons, 21, 427-437.

[9] Little, C.P., Graham, A.J., Karatzas, G., et al. (2005) Outcomes of Total Elbow Arthroplasty for Rheumatoid Arthritis: Comparative Study of Three Implants. The Journal of Bone and Joint Surgery. American Volume, 87, 2439-2448. https://doi.org/10.2106/00004623-200511000-00010

[10] Sanchez-Sotelo, J. (2011) Total Elbow Arthroplasty. The Open Orthopedics Journal, 5, 115-123. https://doi.org/10.2174/1874325001105010115

[11] Aldridge III, J.M., Lightdale, N.R., Mallon, J.W., et al. (2006) Total Elbow Arthroplasty with the Coonrad/Coonrad-Morrey Prosthesis, A 10 to 31 Years Survival Analysis. The Journal of Bone and Joint Surgery. British Volume, 88, 509-514.

[12] Amis, A.A., Dowson, D. and Wright, V. (1980) Elbow Joint Force Prediction for Some Sternous Isometric Reactions. Journal of Biomechanics, 13, 765-775.

[13] Amis, A.A., Miller, J.H., Dowson, D., et al. (1981) Biomechanical Aspects of the Elbow Joint Forces Related to Prosthetic Design. MEP, 10, 65-68. 
[14] Morrey, B.F., Askew, L.J. and An, K.N. (1988) Strength Function after Elbow Arthroplasty. Clinical Orthopaedics, 234, 43-50. https://doi.org/10.1097/00003086-198809000-00010

[15] O’Driscoll, S., An, K.N., Korinek, S., et al. (1992) The Kinematics of the Semiconstrained Total Elbow Prosthesis. The Journal of Bone and Joint Surgery. British Volume, 74, 297-299.

[16] Little, C.P., Graham, A.J. and Carr, A.J. (2005) Total Elbow Arthroplasty A Systematic Review of the Literature in the English Language until the End of 2003. The Journal of Bone and Joint Surgery. British Volume, 87, 437-447.

[17] Rahme, H., Jacobsen, M.B. and Salomonsson, B. (2001) The Swedish Elbow Arthroplasty Register and the Swedish Shoulder Arthroplasty Register: Two New Swedish Arthroplasty Registers. Acta Orthopaedica Scandinavica, 72, 107-112. https://doi.org/10.1080/000164701317323336

[18] Schneeberger, A.G., Adams, R. and Moorey, B.F. (1997) Semiconstrained Total Elbow Replacement for the Treatment of Post-Traumatic Osteoarthrosis. The Journal of Bone and Joint Surgery. American Volume, 79, 1211-1222. https://doi.org/10.2106/00004623-199708000-00014

[19] Throckmorton, T., Zarkadas, P., Sanchez-Sotelo, J., et al. (2010) Failure Patterns after Linked Semiconstrained Total Elbow Arthroplasty for Posttraumatic Arthritis. The Journal of Bone and Joint Surgery. American Volume, 92, 1432-1441. https://doi.org/10.2106/JBJS.I.00145 